\title{
Autonomous Optical Navigation for Spacecraft in Earth Departure Phase
}

\author{
Fu-peng $\mathrm{HE}^{1,2,3}$,Sheng-ying $\mathrm{ZHU}^{1,2,3,{ }^{*}}$ and Ping-yuan $\mathrm{CUI}^{1,2,3}$ \\ ${ }^{1}$ School of Aerospace Engineering, Beijing Institute of Technology, Beijing 100081, \\ China, Hefupeng@bit.edu.cn \\ ${ }^{2}$ Key Laboratory of Autonomous Navigation and Control for Deep Space Exploration, \\ Ministry of Industry and Information Technology, Beijing 100081, China \\ ${ }^{3}$ Key Laboratory of Dynamics and Control of Flight Vehicle, Ministry of Education, \\ Beijing 100081, China
}

Keywords: Optical Navigation, Departure Phase, Observability, Fisher Information Matrix

\begin{abstract}
With the development of navigation technology, the interplanetary missions have made large progress in the past decades. In order to accurately estimate the states of the spacecraft and correctly deliver the spacecraft to the predetermined orbit, deep space autonomous navigation system is investigated. Earlier research has testified that optical navigation technique is a reliable and promising scheme. In this paper, optical navigation system, based on earth and star information, is applied to autonomously update the states of the spacecraft in earth departure phase. To verify the navigation algorithm, observability analysis is performed, the degree of which is quantified by the determinant of Fisher information matrix. An Extended Kalman Filter is used to give the navigation estimate error bound and examine the navigation accuracy of this algorithm in the simulation. The results indicate that the navigation scheme is effective and estimates the position and velocity of the spacecraft accurately.
\end{abstract}

\section{Introduction}

During the past 50 years, NASA, ESA, JAXA and other powerful space countries or organizations have sent plenty of spacecrafts into space to visit other natural bodies in the Solar System. These missions included high speed flyby, rendezvous, orbiting and landing [1]. As one of the challenges in deep space missions, navigation is crucial for successful positioning [2]. Among majority of past deep space missions, this process heavily relied on earth ground tracking station, which, however, significantly increases workload of ground support system.

In order to overcome the limitation, it is necessary to develop a reliable autonomous navigation system that could obtain information about spacecraft location and velocity. As one of the important roles in the autonomous navigation system, optical navigation is extensively used. The key technologies of optical navigation for deep space exploration are developed in Voyager series missions [3]. But the first major attempt to shift navigation from ground-based to onboard operations was made by NASA for the Deep Space 1 mission in 1999 [4]. Afterwards, the Japanese asteroid return mission Hayabusa employed wide angle cameras [5] and Smart-1 of ESA employed the advanced Moon Imaging Experiment camera for onboard autonomous navigation [6]. Moreover, NEAR relied heavily on optical navigation [7].The examples listed above explain the utility of optical navigation to a wide range of missions. 
As the initial phase of deep space exploration mission, earth departure phase plays an indispensable role. If the large orbit errors exist in this phase, the spacecraft has to cost more fuel to correct its orbit in the following phase, which may break the fuel consumption bound and result in the failure of task. Different with the past inertial navigation and ground radio navigation [8], we focus on the optical navigation, aiming to improve the state estimation accuracy with the help of optical observations. In this field, Wu et al. [3] obtained the location and velocity of spacecraft with the information of earth and moon, without considering the navigation observability. As one of the key performance indexes associated with navigation capability, the observability analysis of is important for the design of a navigation scheme [9]. The lower-bound of the error covariance matrix can be estimated by the inverse of Fisher information matrix according to the Cramer-Rao inequality [10]. Therefore, the observability of a navigation system can be analyzed by examining the FIM [11].

Considering the orbit characteristic in the departure phase, this paper imports star and earth information from a star sensor and a ultraviolet sensor into a filter to achieve the optimal state estimation. First, the navigation scheme is presented to introduce information acquisition and processing. Next, the navigation algorithm based on orbital dynamics and measurement model is deduced. Following that, the observability degree is defined and derived using fisher information matrix. Finally, the advantages and accuracy analysis on the basis of angle and sight vector observation is demonstrated by employing an Extended Kalman Filter.

\section{Optical Navigation Scheme}

In this section, the navigation scheme in departure phase using a star sensor and a ultraviolet sensor is given. Earth is an ideal celestial body to be observed. And furthermore, there are enough stars in space to be selected and recognized. So with the obtained multi-celestial information, the scheme is designed to obtain location and velocity of spacecraft in ECI (Earth-centered inertial reference frame).

The navigation software decide which targets to track and what observables to obtain in the camera fields of view by commanding the turntable angle and telescope azimuth and elevations, and setting the focal lengths of cameras. Following that, the camera processing takes the camera data and converts the data into matrix format. Then it is processed by the camera processing algorithms [12] to achieve profile and centroid of earth [1] and star identification. At last, according to the measurement processing data, the location and velocity of spacecraft are deduced with EKF filter.

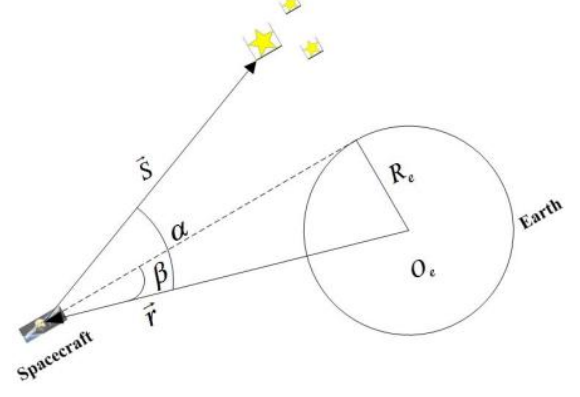

Figure 1. Navigation Principle

Fig. 1 shows the navigation principle. With the data from sensors, the star sight vector $\mathbf{S}$ and geocentric sight vector are obtained. Combining starlight angular distance $\alpha$ and the angle $\beta$ can get measurements, which are calculated as follows: 


$$
\begin{aligned}
& \alpha=\arccos -\mathbf{r} \cdot \mathbf{S} / r . \\
& \beta=\arcsin R_{e} / r .
\end{aligned}
$$

\section{Optical Navigation Algorithm}

Without loss of generality, suppose optical sensor coordinate system and body coordinate system are coincident all the time, in other word, the attitude has been known. Then the navigation algorithm is emerged, which includes state equation and measurement equation. In this part, orbital dynamics equation is reviewed firstly, after that, the measurement model is introduced. At last, Extended Kalman Filter is shown.

\section{Orbit Dynamics Model}

Generally, orbital dynamics equation is considered for spacecraft autonomous navigation as state equation. Perturbation equation in rectangular coordinate system could directly perform location and velocity of spacecraft. So the earth's oblateness perturbation $J_{2}$ is included to improve accuracy. Thus Eq. 3 is obtained.

$$
\begin{aligned}
\dot{\mathbf{r}} & =\mathbf{v} \\
\dot{\mathbf{v}} & =-\mu \frac{\mathbf{r}}{r^{3}}\left[1+\frac{3}{2} J_{2}\left(\frac{R_{e}}{r}\right)^{2}\left(c-5 \frac{z^{2}}{r^{2}}\right)\right]+\Delta \mathbf{F}
\end{aligned}
$$

$\Delta \mathbf{F}=\left[\Delta F_{x}, \Delta F_{y}, \Delta F_{z}\right]$ includes other perturbations such as solar radiation pressure and atmospheric perturbation; $\mu$ is geocentric gravitational constant and $R_{e}$ is radius of earth; $r=\sqrt{x^{2}+y^{2}+z^{2}}$, is geocentric distance for spacecraft in $\mathrm{J} 2000.0$; c is coefficient.

\section{Measurement Model}

Measurement model can be deduced on the basis of angles and unit vector. When the measurement noise is present, the observation model can be characterized by

$$
\mathbf{Z}=\left[\begin{array}{l}
\alpha \\
\beta \\
\mathbf{n}
\end{array}\right]=\left[\begin{array}{c}
\arccos -\mathbf{r} \cdot \mathbf{S} / r \\
\arcsin R_{e} / r \\
\mathbf{r} / r
\end{array}\right]+\left[\begin{array}{c}
v_{\alpha} \\
v_{\beta} \\
v_{\mathbf{n}}
\end{array}\right] .
$$

where $\mathbf{n}$ denotes the geocentric unit sight vector; $\mathbf{v}=\left[\begin{array}{lll}v_{\alpha} & v_{\beta} & v_{\mathbf{n}}\end{array}\right]^{T}$ is called the measurement noise and can be modeled as uncorrelated zero-mean Gaussian noises with the standard deviations of $\sigma_{\alpha}, \sigma_{\beta}, \sigma_{\mathbf{n}}$ to simplify the analysis.

\section{Observability Analysis}

In order to verify stability and reliability of the navigation algorithm, as one of the key performance indexes, observability analysis is introduced. Therefore, the FIM is used for observability analysis.

\section{Fisher Information Matrix}

According to the observation model, the likelihood function of state $\mathbf{X}$ is defined as the joint probability density function of measurements: 


$$
L\left(Z_{1}, Z_{2}, \cdots, Z_{k} \mid \mathbf{X}\right)=\prod_{i=1}^{k} \frac{1}{\sqrt{2 \pi} \sigma_{i}} \exp \left(-\frac{1}{2} \sigma_{i}^{-2}\left\|Z_{i}-h_{i}(\mathbf{X})\right\|^{2}\right) .
$$

The loss function is derived by taking negative of the natural $\log$ of Eq. 5, and retaining only terms dependent on [10], given as

$$
J(\mathbf{X})=\frac{1}{2} \sum_{i=1}^{k} \sigma_{i}^{-2}\left\|Z_{i}-h_{i}(\mathbf{X})\right\|^{2} .
$$

To find estimates of the proper state $\mathbf{X}$ that can minimize the loss function, the fisher information matrix is given as follows:

$$
\mathbf{F}=E\left\{\frac{\partial^{2}}{\partial \mathbf{X} \partial \mathbf{X}^{T}} J(\mathbf{X})\right\}=\sum_{i=1}^{k} \sigma_{i}^{-2} \frac{\partial h_{i}(\mathbf{X})}{\partial \mathbf{X}}\left(\frac{\partial h_{i}(\mathbf{X})}{\partial \mathbf{X}}\right)^{T} .
$$

The relationship between the estimate error covariance and Fisher information matrix can be described as the well-known Cramer-Rao inequality for unbiased estimators[11].

$$
\mathbf{P} \geq \mathbf{F}^{-1} .
$$

In the equation, $\mathbf{P}$ denotes the estimate covariance. Therefore, the observability of the navigation system, which is associated with the estimation performance using multiple measurements, can be analyzed by evaluating the Fisher information matrix of the state. Moreover, the trace of $\mathbf{F}^{-1}$ can be expressed by

$$
\operatorname{tr}\left(\mathbf{F}^{-1}\right)=\sum_{i=1}^{5} 1 / \lambda_{i}
$$

where $\lambda_{1}, \cdots, \lambda_{5}$ are the eigenvalues of $\mathbf{F}$. Thus it can be proved that the larger the eigenvalues of $\mathbf{F}$ are, the smaller the trace of estimate error covariance will be, which means that the observability of the navigation system is stronger. Since the determinant of Fisher information matrix is defined as

$$
\operatorname{det}(\mathbf{F})=\prod_{i=1}^{5} \lambda_{i}
$$

\section{Observability Analysis}

In order to validate the effectiveness of navigation algorithm, a sample is given. Considering the orbit is hyperbolic curve in departure phase, the initial value is given in Table 1 .Thus a $24 \mathrm{~h}$ simulation has been performed to generate the fisher information matrix. 


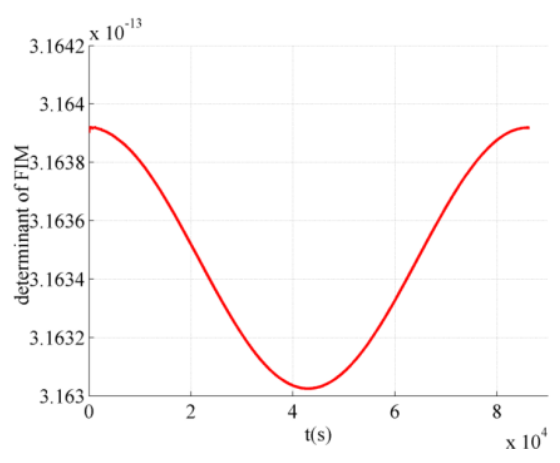

Figure 2. Determinant of FIM

Table 1. Initial value of orbit

\begin{tabular}{ccccc}
\hline $\begin{array}{c}\text { semimajor } \\
\text { axis }\end{array}$ & eccentricity & inclination & argument of perigee & right ascension of ascending node \\
\hline$-3742.1 \mathrm{~km}$ & 5.7796 & $135.395^{\circ}$ & $31.619^{\circ}$ & $89.090^{\circ}$ \\
\hline
\end{tabular}

The determinant of FIM at each time used to measure the navigation performance is illustrated in Fig. 3. Since the state of spacecraft in departure phase is evolved as time goes on, the determinant of FIM also changes. For example, the relatively small value of determinant of FIM appears in the middle period, and one valley occurs. The maximum and minimum determinant of FIM are $3.1639 \times 10^{-13}$ and $3.163 \times 10^{-13}$. Focus on the data depicted, the navigation algorithm meets mission's requirement. So it is easy to draw the conclusion that the algorithm is effective.

\section{Simulation and Results}
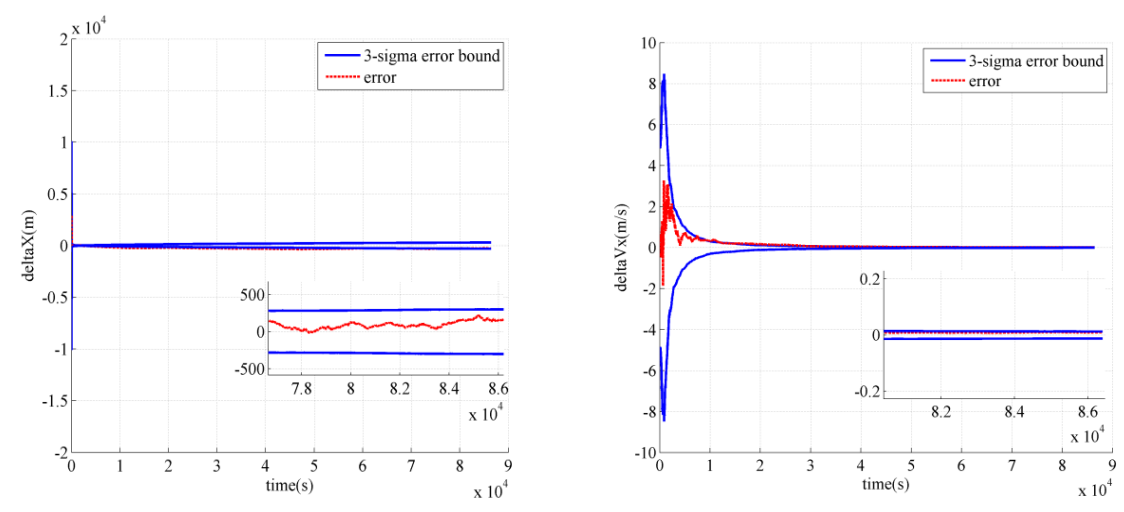

Figure 3. Error of location and velocity in $\mathrm{X}$ direction 

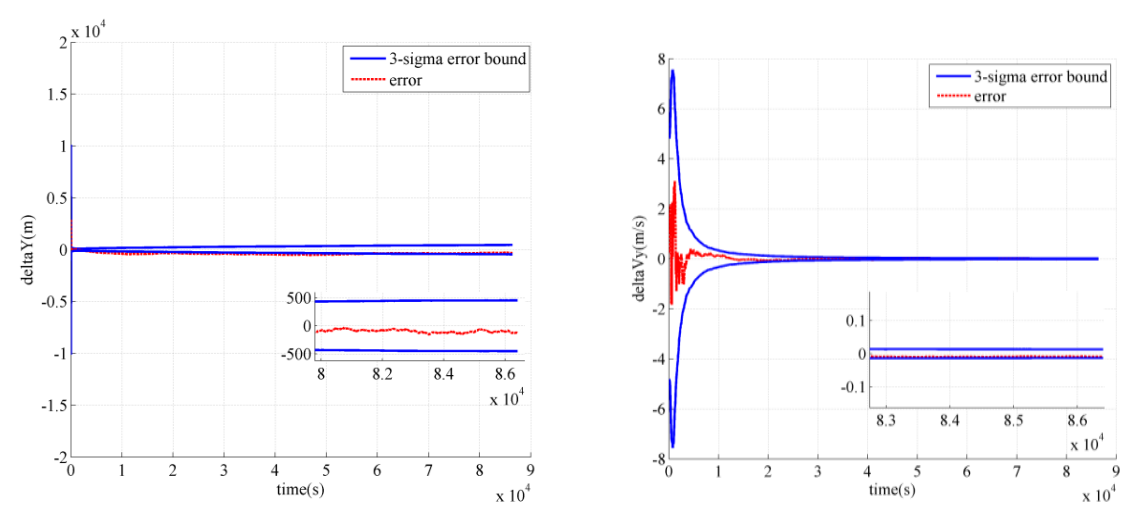

Figure 4. Error of location and velocity in Y direction
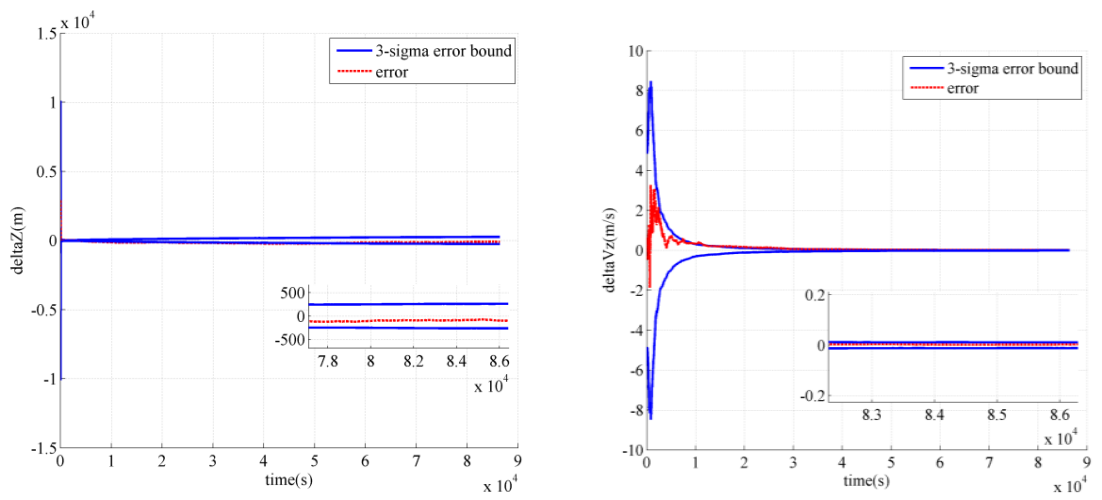

Figure 5. Error of location and velocity in $\mathrm{Z}$ direction

500-time Monte Carlo simulation of navigation system based on EKF is carried out to demonstrate the navigation accuracy. The standard deviation of initial error for location and velocity is set to $10 \mathrm{~km}$ and $5 \mathrm{~km} / \mathrm{s}$ separately. Eq. 3 and Eq. 4 are applied as dynamics and measurement models of the filter.

The $3 \sigma$ uncertainty error bounds curve for three-axis location and velocity are shown with blue lines in Fig. 3, 4, 5, which are recorded in Table 2. Meanwhile, one-time stochastic estimate error results are given in red lines. The thumbnails in each figure provide clear illustrations at stop time.

Table 2. Error bound of the navigation algorithm

\begin{tabular}{lcccccc}
\hline value & $x(\mathrm{~m})$ & $y(\mathrm{~m})$ & $z(\mathrm{~m})$ & $v_{x}(\mathrm{~m} / \mathrm{s})$ & $v_{y}(\mathrm{~m} / \mathrm{s})$ & $v_{z}(\mathrm{~m} / \mathrm{s})$ \\
\hline $\begin{array}{l}\text { error } \\
\text { bound }\end{array}$ & $(-300,300)$ & $(-450,450)$ & $(-240,240)$ & $(-0.008,0.008)$ & $(-0.013,0.013)$ & $(-0.004,0.004)$ \\
\hline
\end{tabular}

Because no information of spacecraft's velocity is provided from measurements, the convergence of velocity estimation is not as quick as location estimation. It can be concluded that the autonomous optical navigation algorithm based on the information of earth and star satisfies the precision requirement in earth departure phase.

\section{Conclusions}

This paper discusses autonomous optical navigation scheme based on earth and star information in earth departure phase. By adopting Fisher information matrix to analyze the observability, the navigation algorithm is verified. In addition, the simulation is demonstrated to show three-sigma error bound. Future work may mainly consider the error effect of image processing on the navigation system. 


\section{References}

[1] Bhaskaran S. Autonomous navigation for deep space missions. In: SpaceOps 2012 Conference, Stockholm, Sweden, 2012.

[2] Martin-Mur T J, Kruizinga G L, Burkhart P D, et al. Journal of Spacecraft and Rockets, 2014, 51(4): 1014-1028.

[3] Wei-ren Wu, Yu-long Tian and Xiang-yu Huang. Journal of Harbin Institute of Technology, 2003, 10(3):343-348.

[4] Riedel J E, Bhaskaran S, Synnott S P, et al. 12th International Symposium on Space Flight Dynamics, 1997, 147(33):470-478.

[5] Kubota T, Hashimoto T, Sawai S, et al. Acta Astronautica, 2003, 52(2-6):125-131.

[6] Polle B. Low thrust missions guidance and navigation: the Smart-1 OBAN experiment results and perspectives.3rd International Workshop on Astrodynamics Tools and Techniques, 2006.

[7] Owen Jr W M, Wang T C, Harch A, et al. Advances in the Astronautical Sciences, 2002, 109:1075-1090.

[8] Riedel J E, Bhaskaran S, Desai S. Using autonomous navigation for interplanetary missions: the validation of Deep Space 1 AutoNav.4th IAA International Conference on Low-Cost Planetary Missions, 2010.

[9] Zheng-shi Yu, Sheng-ying Zhu, Ping-yuan Cui. Acta Astronautica, 2015, 111: 136-145.

[10] Crassidis J L, Junkins J L. Optimal estimation of dynamic systems. CRC press, 2011, 76-81.

[11] Ping-yuan Cui, Zheng-shi Yu, Sheng-ying Zhu, et al. Real-time navigation for Mars final approach using X-ray pulsars. AIAA Guidance, Navigation, and Control Conference and Exhibit, 2013, 5204.

[12] Christian J A, Lightsey E G. Journal of Spacecraft \& Rockets, 2012, 49(2):337-352. 